\title{
A study on the development and implications of low-yield nuclear weapons, focusing on deterrence theory
}

\author{
Sungjoon Moon*
}

\section{ABSTRACT}

This study aims to examine the development status and technical characteristics of low-yield nuclear weapons initiated by the Trump administration, predict the development trend in the Biden administration, analyze strategic implications that have affected the R.O.K.-U.S. Tailored Deterrence Strategy, and seek future countermeasures.

Regarding deterrence theory, low-power nuclear weapons are evaluated as a means of simultaneously expanding deterrence by denial and by retaliation. Additionally, low-yield nuclear weapons can be evaluated as having the capability, communication of nuclear retaliation wills and possibilities, and credibility for these wills and capabilities, which are $3 \mathrm{C}$ elements of deterrence in that they are "possible-use nuclear weapons." Hence, they can be evaluated as highly-applicable deterrence means.

As North Korea's nuclear and missile capabilities have advanced and the U.S. is developing and deploying low-yield nuclear weapons, this article intends to make several suggestions regarding deterrence and response. First, because North Korea's policy to strengthen its tactical nuclear capabilities in 2021 is inevitably closely related to the technical characteristics of the development of low-yield nuclear weapons, it should be evaluated and prepared in connection with this. Second, it is necessary to understand the Biden administration's nuclear strategy regarding the extended deterrence strategy of the U.S. and discuss it closely based on the 5th NPR unveiled in early 2022. Third, to ensure the credibility of the R.O.K.-U.S. tailored deterrence strategy, "multilateral deterrence measures" must be considered at the regional level, including low-yield nuclear weapons.

Keywords : low-yield nuclear weapons, triangular deterrence, Deterrence $3 \mathrm{C}$ factor, tailored deterrence strategy, multilateral deterrence measures

* (First Author) Chosun University, Department of Military Science, Guest professor with professional experience, 24292msj@naver.com, https://orcid.org/0000-0003-3512-5076 


\section{I. 서론}

인류가 개발한 핵무기는 엄청난 파괴력을 갖고 있다. 1945년 미국이 일본 히로시마와 나가사키 에 원자폭탄을 사용한 것이 유일한 실제 핵무기 사용 사례이다. 인류는 핵무기의 엄청난 살상력을 목도하면서 핵무기 실제 사용을 자제하여 왔다. 이른바 ‘공포의 균형'이 핵무기 보유국들의 핵사용 충돌을 막아 온 것이다. 최근 핵무기 사용의 금기(nuclear taboo)를 언제 어디라도 사용가능한 영역 으로 시도하고 있는 흐름을 우리는 예의주시할 필요가 있다.

2018년 미국의 트럼프 행정부에서 공개한 4차 핵태세 검토보고서(NPR: Nuclear Posture Review)는 과거 오바마(Barack Obama) 행정부와 비교하여 세 가지 특징이 있다. 첫 번째는 미국 은 핵무기를 억제전략 구현을 위한 기본적인(fundamental) 조건으로 상정하고 있다는 점이다. 적성 국가(敵性國家)의 핵공격 뿐만 아니라 비핵공격을 억제하는 데에도 핵무기가 효과적이라고 보고 있으며 우방 및 동맹국가의 국가 이익을 지키기 위한 극단적인 환경에서 핵무기를 사용할 수 있음 을 밝혔다. 두 번째로 핵선제 불사용원칙(NFU: No First Use)을 도입하지 않음으로써 핵 선제공격 을 포함한 군사적 옵션을 확대하는 것을 분명히 고려하고 있다는 점이다. 세 번째로 미국이 중국을 러시아에 버금가는 미국 국익에 도전하는 국가로 평가하고 있으며 북한 및 이란의 핵 위협에 대해 서 비교적 상세하게 다루고 있다는 점이다.

미국은 4차 NPR에서 확장억제전략을 구현하기 위한 실질적인 핵전략 강화 방안을 세부적으로 기술하고 있다. 우리가 주목할 것은 핵 3축 체계(triad)의 현대화와 저위력 핵무기(low-yield nuclear weapons) 개발에 대한 구체적인 계획을 제시하였다는 사실이다.1) 특히 기존의 핵무기와 구별되는 저위력 핵무기에 대한 개발을 강화하겠다고 분명하게 밝히고 있다. 미국이 동맹국의 핵무 기가 아닌 재래식 무기에 의한 안보 위협에도 사용가능한 저위력 핵무기로 대응하겠다는 의지를 분명히 밝힌 점은 매우 특이할 만 하다. 2021년 1월 취임한 미국의 바이든(Joe Biden) 대통령은 핵 비확산과 핵축소론을 주장하면서 “핵무기가 국가안보전략에서 차지하는 역할을 줄이겠다”고 선언 한 바 있어 전임 트럼프 행정부가 추진한 저위력 핵무기 프로그램의 축소 또는 폐지가 예상되어 왔다. 그러나 미국의 2022년 회계연도 국방예산안을 보면 저위력 핵무기 예산 항목들이 상당 부분 유지되고 있는 것으로 확인되고 있다. 미국은 저위력 핵무기가 갖고 있는 장점인 정밀성과 대량피 해 최소화와 전 세계에 다양하게 유연성을 갖고 사용가능한 첨단 무기임을 고려 시 저위력 핵무기 개발을 쉽게 포기할 수 없다는 전망이 제기되고 있다.2)

본 연구의 목적은 미국 트럼프 행정부에서 시작한 저위력 핵무기의 개발 현황과 저위력 핵무기

1) 핵 3축 체계는 지상에서 ICBM, 해상에서 SLBM, 공중에서 전략폭격기로 핵무기를 투발하는 체계를 의미하며 저위력 핵무기는 ‘최소 $0.3 \mathrm{kt}$ 까지의 무기위력으로 전략적•전술적 작전 범위의 전개가 가능한 무기이다.

2) 미 바이든 행정부의 핵태세검토보고서(NPR)은 2022년 초에 공개될 예정이다. 이는 5 차 NPR으로 과거 핵의 '전략적 모호성, 기조를 탈피하여 핵선제 불사용원칙(NFU)과 핵을 미국 본토 방어용만으로 사용하는 단일 목적(sole purpose) 사용 여부를 포함할 것인지에 대한 논란이 제기되고 있다. 
의 기술적 특성을 살펴보고 현 바이든 행정부의 개발 추세를 전망한 후에 한미 '맞춤형억제전략 (TDR: Tailored Deterrence Strategy)'에 미치는 전략적 함의를 분석하고 한국의 향후 대응방안을 모색하는 것이다. 이에 본 연구의 방법은 억제 이론을 토대로 미국이 개발 중인 저위력 핵무기 특 성, 북한의 공세적인 핵전략 변화 및 미국의 확장억제전략 발전추세 등의 특정 사실을 정성적으로 살펴보고, 북한의 고도화된 핵능력에 대응할 한미 맞춤형억제전략을 질적으로 평가하고 분석하여 향후 발전방안을 제시하고자 한다.

\section{ㅍ. 이론적 배경}

억제(deterrence)의 사전적 정의는 “감정이나 욕망, 충동적 행위 등을 내리눌러서 그치게 하는 것 또는 정도나 한도를 넘어 나아가려는 것을 억눌러 그치게 하는 것”이다. 이종호는 억제의 정의를 “상대방에게 엄청난 공포를 심어줌으로써 예상되는 결과에 대한 두려움으로 어떤 행위를 하지 못 하게 심리적으로 제지하는 것 또는 상대방에게 보복하겠다고 위협하여 적의 행위를 단념시키는 것"이라고 하였고,3) Synder(1961)는 “공격으로 인한 이익보다 보복으로 인한 피해가 클 것이라는 위협으로 상대방의 공격을 단념하게 하는 것”이라고 하였다.

억제이론(deterrence theory)4)는 '한 국가가 상대국가에 공격을 고려할 시 공격으로부터 얻을 이 익과 피공격국가로부터 받을 보복공격에서 오는 손실을 비교하여 손실이 이익보다 크다고 판단되 면 공격 행위를 단념하게 된다.'는 것이다. 이 억제이론은 미국과 소련 두 강대국 간의 핵전쟁을 막 는 논리적인 근거를 제공해 왔다.5) 이 이론은 군비통제 측면에서 억제에 충분한 군비의 확보 개념 인 '군비경쟁의 안정화(arms race stability)'와 선제공격 동기 제거 개념인 '위기의 안정화(crisis stability)'를 통하여 핵전쟁의 가능성을 감소시키는 역할을 해왔다.6)

통상적으로 억제는 거부적 억제(deterrence by denial)와 보복(응징)적 억제(deterrence by retaliation/punishment)로 구분한다. 거부적 억제는 상대방이 공격을 통해 얻을 수 있는 이익보다 공격에 수반되는 비용과 위험이 훨씬 크다는 것을 인식하여 공격을 사전에 단념시키는 것을 의미 한다. 보복(응징)적 억제는 상대방이 공격 시 훨씬 심각한 피해를 입을 것이라는 2 차 보복 위협을 가함으로써 공격을 억지시키는 것을 의미한다.7) 억제가 효과적으로 작동하기 위해서는 세 가지 요 소가 필요하다. 첫 번째는 상대방의 공격을 거부 또는 보복할 수 있는 능력(capability)을 구비해야

3) 군사학연구회(2014). 군사학 개론. 플래닛미디어, p. 204.

4) 버나드 브로디가 최초로 주장하고, 알버트 홀스테터 등이 이론화하였다(Brodie, 1959; Wohlstetter, 1959).

5) 한용섭(2015). 한반도 평화와 군비통제. 박영사, pp. 11-12.

6) 남만권(2004). 군비통제 이론과 실제. 한국국방연구원, p. 29.

7) 박창권 외(2014). 미국의 확장억제정책과 대북 핵억제전략 발전방향. 국방연구원연구보고서, p. 28. 
한다. 두 번째는 상대방의 공격을 거부 및 보복할 수 있다는 의지를 충분하게 표명하는 의사전달 (communication)이다. 세 번째는 상대방의 공격에 대한 거부 및 보복을 할 수 있는 능력과 의지에 대한 충분한 신뢰성(credibility)을 보장해야 한다. 이를 억제의 3C 요소라고 한다(Haffa, 2018).

미국의 '확장억제(ED: Extended Deterrence)'는 억제 개념을 확대하여 우방 및 동맹국에 대한 공격을 거부하고 응징보복을 할 것이라는 의지 표명으로 적의 공격을 포기하게 만드는 것이다. 확 장억제에서 억제의 $3 \mathrm{C}$ 요소는 우방 및 동맹국의 국가 안전보장에 대한 확신을 갖게 하고, 연루와 방기의 딜레마(abandonment and entrapment dilemma)를 우려시킬 수 있는 필수적인 요소라 할 수 있다.

미국의 ‘맞춤형억제전략(TDR: Tailored Deterrence Strategy)'의 탄생 배경은 아래와 같다. 냉전 시대에 미국은 소련과 함께 보복적 전략핵무기의 상호확증파괴(MAD: Mutual Assured Destruction)에 의한 공포의 균형(a balance of terror)으로 핵전쟁을 억제하는 데에 중점을 두고 억 제이론을 발전시켰다. 즉 양국은 핵무기의 엄청난 파괴력으로 비핵보유국이 핵보유국에 대해 전쟁 을 도발할 가능성은 없다고 보고 상대가 핵보유국인 경우에 전쟁을 억제하는 데에 중점을 두었다. 그러나 1991년 12월 소련이 붕괴하고 탈냉전 시기를 맞이하면서 세계의 전략 환경이 급변하기 시 작하였다. 핵 군비경쟁을 하던 소련의 절대적인 핵위협은 사라졌으나 북한, 이란, 시리아 등이 핵개 발을 시도하고 알카에다에 의한 $9 \cdot 11$ 테러가 발생하였다. 미국은 핵무기의 숫자를 줄여도 안보위 협에 큰 지장이 없다고 판단하였고, 제 3 국에 의한 핵확산과 종교, 민족, 이념 등 비국가 행위자에 의한 테러 위협에 대해 고민을 하기 시작하였다. 미국은 탈냉전 시기에 적합한 억제전략을 모색하 기 시작하였다. 미국이 추진한 첨단 정밀 센서-슈터 체계와 미사일방어체계(MD: Missile Defence) 등의 비핵 체계와 기존의 핵능력을 잘 조합하여 다양한 안보 위협 상황에 적합한 역동적인 맞춤형 억제가 가능하다고 판단하였다. 이러한 배경에서 출발한 맞춤형억제를 동맹국인 한국에 최초로 적 용하기 시작한 것이다. 이 전략은 특정 행위자의 특정 시나리오에 적합한 방책을 적용하여 미국과 우방 및 동맹국들의 사활적 이익을 지키자는 것이었다.8)

지역 핵보유국과 비핵보유국이 혼재한 상태에서 억제구조를 설명하는 데에 ‘삼각억제(triangular deterrence)' 이론이 있다. 이는 확장억제 공약을 제공하는 핵보유국과 핵보유국에 확장억제를 제공 받는 지역 동맹국을 상대해야 하는 국가가 핵보유국을 억제하기 위해 역내 동맹국을 직접적으로 위협하거나 공격 대상화하여 억제를 달성하는 것을 의미한다(Harkavy, 1998). 북한은 핵개발을 한 이유로 미국의 핵무기를 포함한 군사적 위협 때문이라고 주장해 왔다. 이에 북한은 삼각억제 구조 에 있는 미국의 동맹국인 한국, 일본은 물론이고 미국 본토까지 위협할 수 있는 핵과 대륙간탄도미 사일(ICBM: Intercontinental Ballistic Missile), 잠수함탄도미사일(SLBM: Submarine Launched Ballistic Missile)등의 개발을 통해 미국을 위협하면서 미국의 한반도에 대한 군사적 개입을 억제하 고 있다고 보고 있다. 즉, 북한은 한반도를 포함한 동북아 지역에서 한국과 일본을 핵볼모로 삼는

8) 권태영 외(2014). 북한 핵 - 미사일 위협과 대응. 북코리아, pp. 241-262. 
A study on the development and implications of low-yield nuclear weapons, focusing on ... / Sungjoon Moon 117

삼각억제 전략을 사용하여 초강대국인 미국을 주저하게 만들고 있다. 2017년 6월 미 국회청문회에 서 던포드(Joseph F. Dunford Jr.) 합참의장은 북한에 대한 핵선제 공격에 대한 질문에 "미국은 핵 선제 공격 능력을 구비하고 있지만, 한국 수도권에 2,500만 명의 국민이 북한의 위협에 노출되어 있다.”고 언급한 바 있다(Shin, 2018). 현재 북한의 지정학적 및 전략적 환경이 삼각억제 구조로 설 명할 수 있다는 주장에 설득력이 있다고 볼 수 있다. 또한, 이런 삼각억제 개념을 강대국의 핵태세 에 관해 분석하면서 ‘촉매태세(catalytic posture)'라는 개념으로 발전시키기도 하였다(Ahn, 2019; Lee, 2018). 촉매태세는 사활적인 국가이익을 위협받을 때에 강대국의 군사적 혹은 외교적 개입 및 지원을 이끌어내는 것이다. 대표적인 예로 남아프리카공화국이 앙골라의 군사적 위협에 대응하기 위해 핵개발을 시도하면서 미국의 외교적 개입을 끌어낸 것이라고 볼 수 있다(Lee, 2018; Narang, 2014).9)

미국의 저위력 핵무기 관련 선행연구는 2018년 이후부터 이루어지기 시작하였다. 2018년 미국의 $\mathrm{NPR}$ 에 저위력 핵무기의 개발이 포함되면서 국내에서 연구가 진행되기 시작하였다. 김진아는 “2018년 미 핵태세검토보고서의 정책 - 전략적 함의 분석10)”에서 미국의 핵전략 변화와 그 함의를 분석하고 저위력 핵무기의 개발 전망을 언급하였다. 이는 미국의 저위력 핵무기의 개념 정도만 연 구되었고 무기의 실체에는 접근할 수 없었다. 2021년에 들어 조비연은 “미국의 저위력 핵무기와 한 반도에서의 확장억제전략 연구11)"와 “미국의 신형 3종 저위력 핵무기의 기술적 - 전략적 특성과 향 후 전망12)"에서 미국의 저위력 핵무기 개발 및 배치에 대한 분석과 한반도에서의 확장억제전략에 미치는 영향에 관해 연구하기 시작하여 관련 연구진에게 시사성 있고 유의미한 학문적 접근을 가 능하게 해주었다. 이어서 박재완은 “확장억제 신뢰성 제고를 위한 미국의 저위력 핵무기 개발 및 함의”에서 저위력 핵무기를 한국의 억제 - 대응 능력을 강화를 위해 적극적으로 모색할 필요가 있 다고 주장하고 있다. 미국에서 저위력 핵무기 개발이 2018년부터 시작되어 이에 관한 체계적이고 심층적인 연구가 부족한 실정으로 향후 이 분야에 대한 무기체계 발전방향, 이에 대한 각국 핵전략 의 변화추세 등에 관한 연구가 지속될 필요성이 있다.

9) ‘핵태세 최적화 이론'에서 지역핵국들의 핵태세를 제 3 국 개입 촉진을 통해 확전을 위협하는 촉매태세(catalytic posture), 적 핵사용에 대한 보복위협을 하는 확증보복태세(assured retaliation posture), 핵 선제공격을 통해 위협하는 비대칭확전태세 (asymmetric escalation posture) 등 세 가지로 구분하고 있다(Narang, 2014).

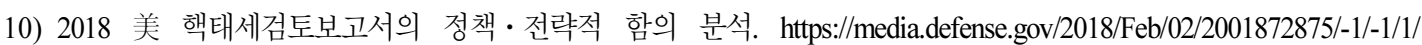
EXECUTIVE-SUMMARY-TRANSLATION-KOREAN.PDF.

11 ) 조비연(2021). 미국의 저위력 핵무기와 한반도에서의 확장억제전략 연구. 한국국방연구원, 국방정책 전문연구시리즈 2021-01. https:/www.kida.re.kr/cmm/viewBoardImageFile.do?idx=29738; 조비연(2021). 미국의 저위력 핵무기와 핵전략 동향. 한국국방연구원, 동북아안보정세분석. https://www.kida.re.kr/frt/board/frtNormalBoardDetail.do?sidx=2184\&idx= $720 \&$ depth $=2$.

12) 조비연(2021). 미국의 신형 3종 저위력 핵무기의 기술적 - 전략적 특성과 향후 전망. 한국국방연구원, 국방논단, 제1864호 (21-32). https://www.kida.re.kr/frt/board/frtNormalBoardDetail.do?sidx=382\&idx=1924\&depth=3\&lang=kr. 


\section{III. 미국의 저위력 핵무기 개발 추세}

\section{1 전략핵무기와 전술핵무기 구분}

핵무기는 통상 전략핵무기(strategic nuclear weapon)와 전술핵무기로(tactical nuclear weapon) 구분한다. 핵무기는 수백 kt 이상의 고위력 핵탄두를 ICBM, SLBM, 전략폭격기 등에 탑재하여 투 발하여 수백만 명의 사상자를 발생시킬 수 있는 전략핵무기(strategic nuclear weapon)와 수십 kt 내외 위력의 핵탄두를 순항미사일, 야포, 어뢰 등의 단거리 투발수단에 탑재하여 수십만 명의 사상 자를 발생시키는 전술핵무기(tactical nuclear weapon)로 각각 구분하였다. 이런 맥락에서 전략핵무 기는 정치적 - 전략적 목표를 달성하기 위해 억제 및 보복수단으로 사용하는 반면에 전술핵무기는 작전적 - 전술적으로 실제 전장에서 사용될 수 있는 무기로 구분하기도 하였다.13) 핵무기의 사용목 적, 표적, 투발수단, 무기위력 등으로 구분하였으나, 사용목적이나 위력의 구분이 모호하다는 측면 이 제기되고 있다. 예컨대, B61 핵폭탄은 무기위력이 0.3kt에서 340kt까지 가능하여 전략핵무기와 전술핵무기의 명확한 구분이 어렵다. 전략핵무기와 전술핵무기의 일반적인 구분은 아래 Table 1 과 같다.

$<$ Table 1> Distinction between strategic and tactical nuclear weapons

\begin{tabular}{c|l|l}
\hline Classifica-tion & \multicolumn{1}{|c|}{ Strategic nuclear weapon } & \multicolumn{1}{c}{ Tactical nuclear weapon } \\
\hline \hline of use & Political $\cdot$ strategic dimension & Operational $\cdot$ tactical dimension \\
\hline Target & $\begin{array}{l}\text { Countervalue } \\
- \text { General public, large city, industrial } \\
\text { facilities, and stockpiles }\end{array}$ & $\begin{array}{l}\text { Counterforce } \\
- \text { Specific military facilities such as } \\
\text { command post }\end{array}$ \\
\hline Weans of fighting power & $\begin{array}{l}\text { Nuclear Triad } \\
- \text { ICBM, SLBM, Strategic bomber }\end{array}$ & $\begin{array}{l}\text { SLCM, torpedo, field gun, } \\
\text { gravity bomb etc. }\end{array}$ \\
\hline Operational reach & Global than hundred $\sim$ thousands kt & $\begin{array}{l}\text { Around tens of kt } \\
* \text { Ambiguous distinction }(\mathrm{B} 61: 0.3 \sim 340 \mathrm{kt})\end{array}$ \\
\hline
\end{tabular}

* Source: adapted from Lee, S. K. (2021)14) and Lieber \& Press (2017)

13) Nonstrategic Nuclear Weapons (CRS Report for Congress). https://www.legistorm.com/reports/view/crs/330685/Nonstrategic Nuclear_Weapons.html.

14) 이상규(2021). 북한의 전술핵 개발 가능성과 핵전략 및 핵 지휘통제 측면에서의 함의. 국방과 기술, 506, pp. 66-73. http://www.dbpia.co.kr/journal/articleDetail?nodeId=NODE10540677. 
A study on the development and implications of low-yield nuclear weapons, focusing on ... / Sungjoon Moon 119

\section{2 저위력 핵무기 개발}

미국은 냉전 이후 전술핵 전력을 일정부분 유지해 왔지만, 독보적인 재래식 전력과 압도적 핵 우 위를 차지하게 된 만큼 재래식 정밀타격 능력을 중심으로 글로벌 억제태세를 구축하면서 전술핵 전력과 교리가 형해화되어 왔다. 2011년 콜린 파월(Colin Powell) 전 미 국무부장관은 "미국은 전술 핵 무기, 특히 핵포병 전력은 문제를 일으키기 쉽고, 현대화하는데 고비용이 소요되며 재래식 무기 체계의 정밀성이 고도화되어 아무런 연결고리가 없다.”고 문제점을 제기한 바 있다. 또한, 2016년 존 하이튼(John E. Hyten) 미 전략사령부(STRATCOM: U.S. Strategic Command) 사령관은 국회 에서 "미국의 핵운용계획이 전략핵과 대량살상파괴 위주로 구성되어 왔다"고 답변한 바 있다. 2018 년 미국 트럼프 행정부는 이를 가리켜 '미국의 역내 억제 전력에 악의적으로 이용 가능한 빈틈 (exploitable gap)이 있음을 지적하면서 NPR을 통해 저위력(low-yield)의 SLBM, 중력폭탄, 잠수함 발사순항미사일(SLCM: Submarine Launched Cruise Missile)의 개발을 선언하였다. 트럼프 행정 부는 핵탄두와 기존 무기체계의 유연한 조합을 통한 다양한 핵능력을 확보하여 맞춤형억제 (tailored deterrence)를 제고시키고자 의도하였다. 2019년 발간된 미 합참의 합동핵작전에 “핵전력 에 필요한 능력(necessary capabilities)으로 다양성, 적응성, 효과성, 대응성, 그리고 생존성이 반드 시 필요하다"고 기술하고 있다(US Joint Chiefs of Staff, 2019).15) 2021년 11월 현재, 미국이 추진하 고 있는 신형 3 종 저위력 핵무기 개발현황은 아래 Table 2와 같다.

$<$ Table 2> Status of the development of a new low-yield nuclear weapons in the U.S.

\begin{tabular}{c|c|c|c}
\hline Classification & $\begin{array}{c}\text { W76-2 Trident- I } \\
\text { SLBM }\end{array}$ & $\begin{array}{c}\text { B61-12 } \\
\text { gravity bomb }\end{array}$ & $\begin{array}{c}\text { W80-4 Tomahawk } \\
\text { SLCM }\end{array}$ \\
\hline \hline Power (kt) & $5 \sim 7$ & $0.3,1.5,10,50$ & Suspense \\
\hline Nuclear warhead & $\begin{array}{c}\text { Low-yield nuclear warhead } \\
\text { W76-2 }\end{array}$ & $\begin{array}{c}\text { Low-yield gravity } \\
\text { bomb B61-12 }\end{array}$ & $\begin{array}{c}\text { W80-4 } \\
\text { improved model }\end{array}$ \\
\hline Means of fighting & $\begin{array}{c}\text { Trident- } \Pi \\
\text { SLBM }\end{array}$ & $\begin{array}{c}\text { fighter jet, bomber } \\
\text { F-15E, F-16C/D, } \\
\text { F-35, B-1B, B-2A, } \\
\text { B-52, B-21, etc. })\end{array}$ & $\begin{array}{c}\text { Tomahawk } \\
\text { SLCM }\end{array}$ \\
\hline Development status & $\begin{array}{c}\text { Development \& deployment } \\
\text { complete in 2019 }\end{array}$ & Scheduled for 2022 & Long-range plan \\
\hline
\end{tabular}

* Source : adapted from Cho, B. Y. (2021).16)

15) Joint Nuclear Operation. "It is imperative that nuclear force capabilities are diverse, flexible, adaptable, effective, responsive, and survivable." https://irp.fas.org/doddir/dod/jp3_72.pdf.

16) 조비연(2021). 미국의 저위력 핵무기와 한반도에서의 확장억제전략 연구. 한국국방연구원, 국방정책 전문연구시리즈 2021-01. 
저위력 핵무기로 첫 번째는 'W76-2형 Trident- II SLBM'이다. 1978년 개발한 전략핵 W76-0핵 탄두(무기위력 100kt)를 2008년에 W76-1핵탄두(무기위력 90kt)로 개량하였는데 이를 2018년에 무 기위력 5 7kt으로 줄이면서 W76-2로 명명하였다. 이 W76-2 핵탄두를 전략 핵탄두의 투발수단인 Trident- IISLBM에 탑재하여 운용하고 있으며 2019년에 개발을 완료하여 실전 배치되었다.

두 번째는 'B61-12중력포탄'이다. 전략 폭격기 또는 전투기에 탑재하여 최대 지하 $100 \mathrm{~m}$ 에 있는 표적까지 타격할 수 있도록 설계되었고, 무기위력은 조정이 가능한 다이얼조정(dial-a-yield) 방식 이며 $0.3,1.5,10,50 \mathrm{kt}$ 4종류가 있다. 과거 $0.3 \sim 300 \mathrm{kt}$ 까지 무기위력을 조정할 수 있는 전술핵폭탄 인 B61을 개량하여 설계하였고 일명 '핵벙커버스터'로 불리고 있다. 2017년 4월에는 F-16 전투기 에 2020년 6월에는 F-15E 전투기에 각각 탑재하여 투발 시험을 성공적으로 완료하였고, 2020년 11 월에는 F-35A 전투기에 탑재하여 첫 투하시험을 하였다.17) 2020년에 개발이 거의 완료되었으나 상 용 커페시터(capacitor)18)가 안정성 문제로 군용으로 개발하고 있어 2022년에 개발 완료될 예정이다.

세 번째로 'W80-4형 Tomahawk SLCM'이다. 퇴역한 토마호크(Tomahawk) 순항미사일을 개량 하여 저위력 핵탄두를 탑재한 SLCM이다. 핵탄두는 장거리 원격무기(LRSO: Long-range Standoff)의 핵탄두로 개발 중인 W80-4 개량형이 탑재될 예정으로 향후 7년 이후 실전 배치할 장 기 프로그램이다.

\section{3 저위력 핵무기의 기술적 특성}

저위력 핵무기는 사용목적이나 투발수단적인 측면에서는 전략핵무기에 가깝고, 위력적인 측면에 서는 전술핵무기에 가깝다. 이러한 구분의 모호성과 사용목적에 대한 논쟁 때문에 '저위력 핵무기 (low-yield nuclear weapon)'는 전략핵무기도 아니고 전술핵무기도 아닌 별도로 구분하고 있다. 즉, 저위력 핵무기는 ‘최소 $0.3 \mathrm{kt}$ 까지의 무기위력으로 전략적 - 전술적 작전 범위의 전개가 가능한 다양 성과 유연성을 구비한 첨단 핵전력’이라고 정의하고 있다.19)

저위력 핵무기는 기존의 전략과 전술 핵무기와 비교하여 다른 특성이 있다. 첫 번째, 저위력 핵 무기는 투발수단의 다양성을 보유하고 있다. 저위력 핵무기는 SLBM, 장거리 전략폭격기, 전투기 (F-15,16,35) 등에도 탑재가 가능하다. W76-2형 Trident- II 잠수함탄도미사일(SLBM)은 무기위력 이 5 7kt으로 무기위력 면에서 전술핵으로 분류할 수 있으나 핵잠수함에 탑재하여 작전 수행 범 위가 세계 전역으로 확장되었다. 미국은 2019년 말 오하이오급 핵잠수함 USS 테네시함 (SSBN-734)에 저위력 핵무기를 탑재하여 대서양 지역에서 '전략적 억제작전(strategic nuclear

17) Newswise(2020. 11. 23). Flight Tests to Show B61-12 Will Work on Air Force's Newest Fighter Jet. https://www.newswise.com/articles/flight-tests-to-show-b61-12-will-work-on-air-force-s-newest-fighter-jet.

18) 폭발유도장치의 전력 제공을 위한 축전기(condenser) 부품.

19) 조비연(2021). 미국의 저위력 핵무기와 한반도에서의 확장억제전략 연구. 한국국방연구원, 국방정책 전문연구시리즈 2021-01. https://www.kida.re.kr/cmm/viewBoardImageFile.do?idx=29738. 
deterrence operation)'을 수행한 바 있어 저위력 핵무기를 기존의 전술핵무기로 구분할 수도 없는 실정이다. 저위력 중력폭탄 B61-12도 장거리 전략폭격기 $(\mathrm{B}-1 \mathrm{~B}, \mathrm{~B}-2 \mathrm{~A}, \mathrm{~B}-52, \mathrm{~B}-21)$ 뿐만 아니라 $\mathrm{F}-15, \mathrm{~F}-16, \mathrm{~F}-35$ 전투기에도 탑재하여 작전 목적에 따라 다양하게 운용될 수 있으며 최근 극초음 속 미사일 개발에 W76-2 저위력 핵탄두를 탑재하는 방안도 고려되고 있는 것으로 알려져 있다 (Cummings, 2020).

두 번째, 저위력 핵무기는 고도의 정밀성을 보유하고 있다. 미국은 $9 \cdot 11$ 테러 이후 지하 깊숙이 있는 시설과 은거해 있는 핵심 인물을 동시에 파괴 및 살상할 수 있는 무기체계 개발에 집중해 왔 다. B61-12 저위력 핵벙커버스터는 전폭기나 전투기에서 투하되어 지하 시설물을 함몰하여 파괴하 는 'earth penetrator' 기능을 수행할 수 있다. 또한, 꼬리 날개를 장착하여 목표지점까지 날아갈 수 있고 내부에 범세계위치확인시스템(GPS: Global Positioning System)을 장착하여 정밀폭격이 가능 한 핵유도폭탄(nuclear-guided bomb)이다.20) 저위력 핵탄두 W76-2는 타격 오차 범위도 90m 이하 이고 슈퍼신관을 이용한 B61-12와 같이 핵벙커버스터로 사용 가능해졌다는 평가를 받고 있다.21)

세 번째, 저위력 핵무기는 핵무기 사용의 가능성을 증대시켜 주고 있다. 그동안 핵폭발 시 대량 인명피해, 방사선과 낙진으로 인한 2차 피해 등으로 핵사용은 극히 제한되었다. 그러나 2017년 미 국 국방부 ‘위험예측 및 평가(HPAC: Hazard Prediction and Assessment Capability)' 시뮬레이션 프로그램을 적용한 연구결과, 북한 내 5개소의 목표물을 파괴하기 위해 $475 \mathrm{kt}$ 무기위력을 갖는 W88 전략핵탄두가 탑재된 Trident- II 1발 폭발 시 남북한에서 200 300만 명의 사상자가 발생하 는 반면에 저위력 핵무기 B61-12형 1발을 투하 시 100명 미만의 사상자가 발생하는 것으로 피해 예측한 바 있다(Lieber \& Press, 2017). 이는 저위력 핵무기가 재래식 정밀유도무기와 유사한 정도 로 인명피해를 획기적으로 줄이면서 핵사용 금기(nuclear taboo)를 극복할 수 있는 가능성을 보여 주고 있다.

\section{4 저위력 핵무기 개발 전망}

2021년 3월 공개한 미국의 '잠정 국가안보전략지침22)(INSSG: Interim National Security Strategic Guidance)'에는 “미국은 국가안보전략에서 핵무기의 역할을 줄여나가는 조치를 취해 나 갈 것이다.”고 밝혔다. 이를 토대로 미국의 바이든 행정부에서 저위력 핵무기 개발 축소가 예상되 었다. 그러나 2021년 5월 미국 국방부가 의회에 제출한 2022년 국방예산을 살펴보면, 저위력 핵무 기 예산이 전년 대비 상당 부분 유지되고 있는 것을 알 수 있다. Table 3과 같이 저위력 핵순항미사

20) 글로벌이코노믹(2020.6.10.). [G-Military] 미군이 투하시험 성공한 B61-12는? 폭발력 50kt의 핵무기. https://cmobile.g-enews.com/view.php?ud=202006100848259555c5557f8da8_1\&md=20200610092243_S

21) 세계일보(2021). 읏 사용가능 저위력 핵무기 확충 전망. http://m.segye.com/view/20210811514708

22) https://www.whitehouse.gov/wp-content/uploads/2021/03/NSC-1v2.pdf 
일 관련 예산은 전년대비 1천만 달러(약 113 억원) 신규 편성되었고, 저위력 중력폭탄 관련 예산은 전년 8.16억 달러에서 7.72 억 달러로 $0.5 \%$ 소폭 삭감되었다.23)

$<$ Table 3> The current status of budgeting for low-yield nuclear weapons in the fiscal year 2022 of the Biden administration in the U.S.

\begin{tabular}{c|c|c|c|c}
\hline Classification & Nuclear warhead & FY 2021 & FY 2022 & Change \\
\hline $\begin{array}{c}\text { Low-yield } \\
\text { SLCM }\end{array}$ & W80-4 & - & $\$ 10$ million & $+\$ 10$ million \\
\hline $\begin{array}{c}\text { Low-yield } \\
\text { gravity bomb }\end{array}$ & B61-12 & $\$ 8.16$ billion & $\$ 7.72$ billion & $\begin{array}{c}-0.5 \% \text { cut } \\
(-\$ 0.44 \text { billion })\end{array}$ \\
\hline
\end{tabular}

* Source : adapted from FY2022 Congressional Budget Request in U.S. Department of Energy (2021).

U.S. Department of Energy (2021). FY2022 Congressional Budget Request, 1-10.

미국의 바이든 대통령과 민주당은 핵비확산과 핵축소를 지지하는 외교정책을 중시해 왔다. 그들 은 핵확산금지조약(NPT: Nuclear nonproliferation Treaty) 국제레짐에 의한 글로벌 핵비확산과 핵 보유국의 핵군축을 지지하고, 이란 핵합의(JCPOA: Joint Comprehensive Plan of Action)24)를 중시 하며 북한에 이란의 핵합의 모델을 적용하자고 주장하기도 하였다.25) 2021년 1월 26일 바이든 대통 령은 러시아의 푸틴 대통령과 신전략무기감축협정(New START: New Strategic Arms reduction Talks)을 재연장하기로 합의하였다. 그러나 이 합의는 1,500 개의 전략 핵탄두와 700 기의 핵투발수 단에 대해서만 제한하고 있을 뿐 중 - 단거리 전술핵무기와 저위력 핵무기는 포함되어 있지 않다. 바이든 행정부도 중국과의 패권 경쟁이 치열해지고 북한의 핵 고도화, 이란과의 핵합의 복원 등을 앞두고 핵을 토대로 한 억제의 필요성을 인정할 수밖에 없을 것으로 보이며 저위력 핵무기에 대한 개발 및 배치도 전면 폐지보다는 현상유지 내지는 부분적 감소 수준에서 유지할 것으로 전망된다.

23) 조비연(2021). 미국의 저위력 핵무기와 한반도에서의 확장억제전략 연구. 한국국방연구원, 국방정책 전문연구시리즈 2021-01.

24) JCPOA는 2015년 7월 14일 이란과 P5+1(미국, 러시아, 프랑스, 영국, 중국 + 독일)간 이란의 핵프로그램 해결을 위한 다자합의이다. 그러나 2018년 5월 8일 미국 트럼프 대통령이 독자적으로 탈퇴 결정을 선언하였고 이란의 핵문제는 다시 봉착상태에 있다.

25) 전봉근(2021). 바이든 행정부의 북핵협상 시 예상쟁점과 대응전략. 외보안보연구소. http://www.ifans.go.kr/knda/ifans/kor/ pblct/PblctView.do;jsessionid=g-QazL8D-QZsjMN6YChCcFvP.public21?csrfPreventionSalt=null\&sn=\&bbsSn=\&mvpSn=\&s earchMvpSe=\&koreanEngSe=KOR\&ctgrySe=\&menuCl=P01\&pblctDtaSn=13741\&clCode=P01\&boardSe= . 


\section{IV. 억제이론 면에서 전략적 함의 분석}

\section{1 북한의 핵전략 변화 추세 분석}

북한은 2012년 4월 13일 개정헌법에서 “김정일 동지는 우리 공화국을 불패의 정치사상 강국, 핵 보유국, 무적의 군사강국으로 전변시켰다”고 하면서 처음으로 핵무장을 기정사실화하였다. 이어 2013년 4월 1일 ‘핵보유국법’을 채택하고 핵전략을 처음으로 공개하였다. 법 2조 핵무기의 용도에 서 “핵무장력은 우리 공화국에 대한 침략과 공격을 억제 및 격퇴하고, 침략의 본거지에 섬멸적인 보복타격을 가하는데 있다”고 하면서 핵무기는 상대방의 공격에 대한 억제용이고 억제 실패 시에 는 보복용임을 분명히 하였다. 2013년 3월 27일 북한 인민군 최고사령부 명의 성명을 통해 “대남 군사적 행동은 우리의 자주권 수호를 위한 강력한 핵선제 타격을 포함한다"고 주장하였고, 2016년 3월 7일 북한 국방위원회 성명에서 “적들의 합동군사연습이 우리 공화국의 자주권에 대한 노골적 인 핵전쟁 도발로 간주된 이상 우리의 군사적 대응도 더욱 선제적이면서 공격적인 핵타격전이 될 것이다.”라고 하면서 핵선제공격을 공언한 바 있다.26) 북한은 2021년 1월 5일부터 12 일까지 실시된 조선노동당 제8차대회 당중앙위 총화보고에서 “핵기술을 더욱 고도화하면서 핵무기의 전술무기화, 소형경량화를 보다 발전시켜 현대전에서 작전 임무의 목적과 타격 대상에 따라 각이한 수단으로 적용할 수 있는 전술핵무기들을 개발하고 초대형 핵탄두 생산도 지속적으로 추진함으로써 핵위협 이 수반되는 조선 반도지역에서의 군사적 위협에 주동성을 유지하며 철저히 억제하고 통제관리 할 수 있게 하여야 한다"고 하였다.27) 북한의 당대회 보고문에 포함된 전술핵 강화 방침과 2021년 계 속되는 단거리탄도미사일, 순항미사일 발사 등은 유사시 한반도 전구 내 핵을 이른바 실전에서 즉 각 활용전력으로 검토하고 있다고 볼 수 있다(Hwang, I. D., 2021).

북한은 핵무기의 소형화, 투발수단의 다양화 등 전술핵 사용의 문턱(threshold)을 최대한 낮춤으 로써 재래식 전력의 열세를 상쇄(countervailing)시키고, 전술핵 사용 후에도 대량의 전략핵을 보유 함으로써 미국의 전략핵 응징보복을 피할 수 있는 핵전략을 모색하고 있는 것으로 보인다. 특히, 북한이 제 8차 당대회에서 전술핵 개발 강화 방침에 더하여 언급한 ‘중장거리순항미사일'과 핵 투 발수단으로 후보군으로 가능한 $\mathrm{KN}-23, \mathrm{KN}-24, \mathrm{KN}-25^{28)}$ 등의 전술유도무기인 단거리탄도미사일 (SRBM: Short Range Ballistic Missile)이다.29) 이러한 투발수단이 핵무기 투발에 사용되었다는 직

26) 전봉근(2020). 비핵화의 정치. 명인문화사, pp. 295-302.

27) 홍민 외(2021). 북한 조선노동당 제8차대회 분석. 통일연구원, pp. 26-32.

28) 미국은 북한 미사일 명칭 부여를 식별된 순서에 따라 ‘KN(Korea North) 숫자'로 명명하고 있다. 북한은 우주발사체는 백두산, 광명성, 은하 등으로 전략군 화성포병부대에서 운용하는 미사일은 ‘화성’으로 SLBM은 ‘북극성’으로 각각 명명하고 있다.

29) 북한이 집중개발하고 있는 전술유도무기인 SRBM은 KN-23(신형전술유도탄, 북한판 이스칸데르 개량형인 고체연료 단거리탄도미사일), $\mathrm{KN}-24$ (화성-11 개량형, 북한판 에이테킴스라고 부르는 고체연료 단거리지대지미사일), $\mathrm{KN}-25$ (단거 
접적인 증거는 아직 없지만, 향후 지속적으로 이 분야에 대한 연구개발을 시도할 것으로 전망하고 있다.30) 2021년 들어 북한이 당대회에서 전술핵 역량강화를 언급하면서 개발에 박차를 가하는 데 에는 기술적 도전 요소들이 있지만 일단 성공을 한다면 전략적 - 전술적으로 효율적인 레버리지를 확보할 수 있을 것이다(Hwang, H. P., 2021). 북한의 전술핵 역량 강화는 필연적으로 저위력 핵무 기 개발의 기술적 특성과 밀접한 연관이 있어 이를 연계한 평가가 필요하다.

삼각억제 구조 측면에서 북한은 유사시 미국의 한반도 개입을 저지하기 위해 한국과 일본을 동 시에 억제해야 한다. 이를 위해 북한은 한미, 미일연합전력에 대한 비대칭확전 역량과 미국에 대한 확증보복 역량의 동시 구비를 강요받고 있다. 즉 북한의 삼각억제 구조는 북한의 핵 - 미사일 위협 에 대한 동맹국에 대한 확장억제(extended deterrence)와 본토억제(central deterrence)를 동시에 대 비해야 하는 미국의 억제구조와 유사하다고 볼 수도 있다(Sohn, 2015). 향후 북한은 핵무기 사용에 따른 대량 인명살상에 대한 정치적 - 도덕적인 책임을 우회하면서 전략적-전술적으로 언제 어디 서라도 요망하는 표적에 공격이 가능한 저위력 핵무기의 개발에 관심을 갖게 될 것이다.

\section{2 저위력 핵무기의 전략점 함의}

저위력 핵무기는 거부적 억제(deterrence by denial)와 보복적 억제(deterrence by retaliation)를 동시에 확대할 수 있는 수단으로 평가받고 있다. 국가의 거부적 억제력의 효과성과 신뢰성을 보장 하는 핵심은 바로 즉응성과 사용가능성에 있다. 기존의 핵무기는 대량살상에 따른 정치적 - 도덕적 비난에 대한 부담으로 금기되었다. 그러나 저위력 핵무기는 인명 피해 최소화와 정밀성을 기반으로 핵위기 및 급변사태와 같은 높은 수준의 군사적 긴장 상태 하에서 신속하게 거부 의지와 신뢰성을 높일 수 있는 수단이 될 수 있다. 보복적 억제 측면에서도 저위력 핵무기는 효과적인 수단이 될 수 있다. 저위력 핵무기는 무기위력 면에서 기존의 전술핵무기에 비해 약하지만 유사시 적 수뇌부, 핵 심적인 군사시설 등에 대한 외과적 수술(surgical strike)을 가능하게 하여 실질적인 2차 보복의 신 뢰성을 증대시킬 수 있다. 저위력 핵무기는 제한된 파괴력을 갖고 있으나 대량살상, 방사선 및 낙진 피해 등을 최소화하면서 사용가능한 핵무기로 거부적 억제와 응징적 억제력을 확대할 수 있는 새 로운 선택방안으로 주목받고 있다. 저위력 핵무기는 '사용가능한 핵무기'라는 점에서 억제의 $3 \mathrm{C}$ 인 사용가능한 능력(capability), 핵보복 의지와 가능성의 의사전달(communication), 이러한 능력과 의 지에 대한 신뢰성(credibility)이 확보되어 억제의 수단으로 활용 가치가 높다고 볼 수 있다(Park, 2021).

둘째, 미국의 확장억제전략 차원에서 한반도에 미치는 영향을 예의 주시할 필요성이 있다. 미국

리탄도미사일의 일종인 초대형방사포)이다.

30) 이상규(2021). 북한의 전술핵 개발 가능성과 핵전략 및 핵 지휘통제 측면에서의 함의. 국방과 기술, 506, pp. 66-73.

http://www.dbpia.co.kr/journal/articleDetail?nodeId=NODE10540677 
이 확장억제를 동맹국에 약속한 전략적 목적은 두 가지이다. 우선적으로 지역안보를 통한 자국의 이익을 확대하는 것이다. 유럽, 아시아 지역의 우방 및 동맹 국가들에게 핵우산과 전쟁 억제력을 제공하여 지역 안보 협의체를 공고히 하여 미국 본토에 대한 안보위협이 미치지 않게 하여 외교적, 경제적, 군사적 이익을 확대하는 것이다. 다음으로 글로벌 핵비확산 정책을 통한 자국의 안보 증대 이다. 비핵국가에 대한 원자력의 평화적 이용은 보장하되 핵무장은 철저히 억제하여 미국의 안보위 협을 안정적으로 관리하고자 하는 것이다.31) 이러한 측면에서 동북아 지역 내 미국의 확장억제 전 략에 하나의 선택지가 될 수 있는 저위력 핵무기의 개발 및 배치를 현 미국의 바이든 정부는 고민 하지 않을 수 없을 것이다. 저위력 핵무기의 장점인 전략적 - 전술적 투발수단의 다양화, 정밀성을 증대하여 요망하는 표적에 핀셋식 타격 가능, 핵무기 사용으로 인한 피해 최소화 등으로 세계 전 지역에서 유연하고 사용가능한 핵무기의 활용 여부를 예의 주시할 필요가 있다. 당장 미국이 한반 도에 저위력 핵무기의 배치 및 운용할 가능성은 적어 보인다. 북한과 중국의 반대는 물론 한국 내 부의 반발도 심할 수 있기 때문이다. 특히 미국은 20여 년 동안 재정적 - 군사적 지원을 아끼지 않 았던 아프가니스탄에서 철군하고 중국에 대한 견제와 압박에 집중하고 있다. 미국은 쿼드(QUAD), 오커스(AUKUS)32) 등 다자 동맹을 통해 중국을 전방위적으로 압박하고 있다. 한반도에서의 핵 위 기가 고조될 경우에 이미 개발과 배치가 완료된 W76-2형과 같은 저위력 핵무기는 미국이 확장억 제력을 신속하게 현시할 수 있는 있을 것이다. 따라서 향후 저위력 핵무기의 개발 추세를 주목하고 미국의 확장억제력에 미치는 영향을 분석하여 대응해 나가야 할 것이다.

\section{3 한미 맞춤형억제전략 발전방안}

2013년 10월 2일 제45차 한미안보협의회의(SCM: Security Consultative Meeting)에서 한미 국 방부장관은 북한의 핵 - 미사일 위협에 대응하여 한반도 상황에 최적화된 한미 공동의 대응전략인 ‘맞춤형억제전략'에 서명한 바 있다. 이는 미국이 우방 및 동맹국에 제공하는 일반적인 ‘확장억제' 개념보다 진일보한 전략 개념으로 북한이 핵무기 사용을 위협하는 단계부터 핵무기를 직접 사용하 는 단계까지 한미 양국이 보유한 군사적 - 비군사적인 모든 수단과 방법으로 공동 대응하는 방안이 포함되어 있다.33)

한미 맞춤형억제전략이 성공적으로 작동하기 위한 전제조건이 있다. 첫째, 북한의 핵 - 미사일 위협의 실체를 정확히 인식해야 한다. C4ISR34)자산과 인간정보(HUMINT: Human Intelligence)

31) 조비연(2021). 미국의 저위력 핵무기와 한반도에서의 확장억제전략 연구. 한국국방연구원, 국방정책 전문연구시리즈 2021-01.

32) QUAD는 미국, 일본, 호주, 인도의 4자간 안보협의체이며 중국을 견제하는 공동의 목적을 가진다. AUKUS는 호주, 영국, 미국의 3 자 안보협의체로 최근 호주의 핵잠수함 개발 지원을 목표로 하고 있다.

33) 대한민국 국방부 (2020). 2020 국방백서. 국방부, pp. 59-60. https://www.mnd.go.kr/user/mnd/upload/pblictn/ PBLICTNEBOOK_202102040549325290.pdf 
자산의 효율적인 결합을 통한 북한 위협의 실체를 정확히 직시하는 것이 우선적으로 필요하다. 둘 째, 억제 능력의 구비로 군사적 - 비군사적 수단과 방법을 망라한 적을 압도하는 능력을 구축할 필 요가 있다. 외교 - 정보 - 군사 - 경제적 $(\mathrm{DIME}) 35$ ) 요소 등이 적절한 예가 될 수 있을 것이다. 셋째, 동맹의 신뢰와 확고한 의지이다. 제일 중요한 전제조건이라 할 수 있다. 이는 억제를 할 수 있는 물리적 수단이 한반도에 가시적으로 존재해야 하고, 미국이 한국을 지키겠다고 하는 의지가 있어야 하며 동맹이 연습과 훈련을 통한 역량을 현시하는 것이 중요하다.36)

한미 맞춤형억제전략은 그동안 북한의 핵 - 미사일 위협에 대응하여 한반도 안보를 안정적으로 관리하는데 기여했다고 인정하는 긍정적인 시각도 있지만, 여전히 우려하는 바가 존재한다. 가장 핵심적인 사항은 맞춤형 억제전략 보장의 신뢰성에 관한 의구심이다(Hwang, J. W., 2021). 한국이 필요로 할 때 미국의 지원은 필요충분할 것인가에 대한 의문이다. 북한의 핵능력은 미국의 핵심동 맹국인 일본과 미국 본토까지 위협하면서 한국 안보를 전략적 거래의 대상으로 활용할 수 있는 공 간을 갖게 되었다는 사실이다. 북한은 미국이 내재적으로 갖고 있는 이익의 비등가성을 저울질하면 서 이를 적극 활용하고자 할 것이다. 즉 미국이 워싱턴을 포기하면서 서울을 지켜낼 것인가에 대한 물음이 맞춤형억제전략에 대한 의구심을 촉발시키는 근본적인 이유이다. 이에 따라 한반도에 전술 핵 배치, 나토식 핵공유 방안 등이 꾸준히 제기되고 있다. 북한의 공세적인 핵태세 구축과 고도화된 핵역량을 고려하여 한미동맹 차원에서 통합된 맞춤형억제전략의 보완이 필요한 시점이다.

한반도의 지정학적인 구조에 기인한 삼각억제의 구조에서 기인하는 북한의 핵 - 미사일 위협에 효과적으로 대응하기 위한 지역 차원의 ‘다자 억제 방안’을 진지하게 검토해야 한다(COCHAIRS, 2021). 예를 들어 미국이 개발 및 배치하고 있는 저위력 핵무기의 한반도 배치가 제한된다면 일본 또는 미국의 괌에 배치하거나 핵잠수함의 전진 배치 등 한반도 역내 억제력을 제고하는 방안을 고 려할 수 있을 것이다. 미국의 핵전력과 우방 및 동맹국의 재래식 전력을 통합하여 운용할 수 있는 지역 차원의 안보 플랫폼을 구축하는 시도를 추진할 수 있다.

\section{V. 결론 및 논의}

미국이 2018년 개발에 착수한 저위력 핵무기는 최소 0.3kt까지의 무기위력을 갖고 고도의 정밀성 으로 인명피해를 최소화하며 사용가능한 첨단 핵전력이다. 저위력 핵무기는 전략적 - 전술적 투발 수단이 다양하고, 요망하는 표적, 특히 지하 시설물에 대한 정밀한 파괴 효과와 대량 인명피해를 방지할 수 있다는 특별한 장점을 갖고 있다. 억제이론 측면에서 저위력 핵무기는 거부적 억제와 보

34) C4ISR : Command, Control, Communications, Computers, Intelligence, Surveillance and Reconnaissance

35) DIME : 외교(Diplomacy), 정보(Intelligence), 군사(Military), 경제(Economy)

36) 권태영 외(2014). 북한 핵 - 미사일 위협과 대응. 북코리아, pp. 239-246. 
복적 억제를 동시에 대비할 수 있는 수단으로 평가받고 있다. 저위력 핵무기는 '사용가능한 핵무기' 라는 점에서 사용가능한 능력(capability), 핵보복 의지와 가능성의 의사전달(communication), 이러 한 능력과 의지에 대한 신뢰성(credibility)이 확보되어 억제의 수단으로 활용 가치가 높다.

북한은 핵 - 미사일 능력을 고도화하면서 핵전략을 전쟁 억제용, 방어용, 보복용, 선제타격용 등 으로 확대하고 있다. 미국은 트럼프 행정부 이래로 중국, 러시아는 물론 북한, 이란 등의 전방위적 위협에 대응하기 위해 실제 전장에서 사용가능한 저위력 핵무기를 개발 및 배치하고 있다. 북한의 핵 - 미사일 위협을 직접적으로 받으면서 미국과의 맞춤형억제전략에 전적으로 의존하는 한국의 전쟁 억제와 대응 측면에서 몇 가지 발전적 제안을 하고자 한다.

첫째, 2021년 북한의 전술핵 역량 강화방침에 주목해야 한다. 전술핵무기 개발은 필연적으로 향 후 저위력 핵무기 개발과 연계되어 있음을 직시해야 한다. 북한은 한반도에서 작전적 - 전술적 목 적 달성에 필요한 사용가능한 전술 및 저위력 핵무기를 개발하고자 할 것이다. 북한은 핵무기의 소 형화, 다종화는 물론 ‘중장거리순항미사일'과 $\mathrm{KN}-23, \mathrm{KN}-24, \mathrm{KN}-25$ 등 3종의 전술유도무기인 단 거리탄도미사일(SRBM) 등 핵 투발수단의 다양화를 지속적으로 시도하고 있다. 이에 북한의 핵능 력 확장과 핵전략 변화 및 발전추세를 객관적으로 진단하고 억제 및 대비해 나가야 할 것이다.

둘째, 북한의 핵 - 미사일 위협에 대응하기 위해 한국은 한미 맞춤형억제전략을 보다 신뢰성을 제고할 수 있는 방향으로 발전시켜 나갈 필요성이 있다. 미국의 바이든 행정부는 국가 대전략, 지역 안보전략, 동맹전략의 큰 틀 안에서 확장억제전략을 추진할 것이다. 이를 위해 우리는 미국 바이든 행정부의 핵에 대한 인식, 전략-전술핵무기와 저위력 핵무기를 포함하는 핵전력의 역할과 운용방 안, 북한의 주요 위협과 핵심 억제 대상, 비핵전력의 추가적인 역할 등에 관해 심층적으로 분석하여 대응전략을 마련하고 긴밀하게 협의하여 전쟁을 억제하고 유사시에는 전승을 보장할 수 있도록 해 야 할 것이다.

셋째, 한미 맞춤형 억제전략의 발전적인 방안으로 한반도의 지정학적인 구조에 기인하는 북한의 핵 - 미사일 위협에 효과적으로 대응하기 위한 지역 차원의 ‘다자 억제 방안'을 진지하게 검토해야 할 것이다. 미국이 개발 및 배치하고 있는 저위력 핵무기의 한반도 배치가 제한된다면 일본 또는 미국의 괌에 배치하여 한반도 역내(域內) 핵억제력을 제고하는 방안도 고려할 수 있을 것이다. 나 아가 미국의 핵전력과 우방 및 동맹국의 재래식 전력을 통합하여 운용할 수 있는 지역 차원의 안보 플랫폼을 구축하는 시도를 점진적으로 진행해야 할 것이다.

미국 트럼프 행정부 시절에 개발에 착수한 저위력 핵무기를 현 바이든 행정부에서 어떻게 정책 방향을 취할 것인가에 대해 전 세계가 주목하고 있다. 2022년 초에 나오게 될 5 차 NPR을 주목하지 않을 수 없다. 유럽연합(EU: European Union) 및 한국, 일본 등은 핵선제불사용원칙과 핵의 단일 목적사용 등이 NPR에 포함 여부를 두고 우려를 하고 있다. 동맹관계에서 '연루와 방기’라는 태생 적인 안보 딜레마가 존재할 수밖에 없다. 핵무장국인 미국이 맞춤형억제전략으로 핵우산을 제공하 겠다는 확약을 하더라도 한국 입장은 '방기'의 우려를 나타낼 수밖에 없을 것이다. 향후 한반도 역 
내에서의 미국의 저위력 핵무기 활용은 미국의 전략적 목표, 북한과 중국의 위협 인식과 반발, 한미 동맹의 관계, 언론과 국민 여론 등을 고려하여 맞춤형억제전략에 포함시켜 발전시켜 나가야 할 것 이다.

본 논문에서 다루지 못한 몇 가지 부분에서 지속적인 후속연구가 필요하다고 본다. 미국의 저위 력 핵무기 개발에 따른 한국의 억제 대응전략, 2022년 미국의 5 차 NPR를 토대로 저위력 핵무기의 발전추세를 추적하고 한미 맞춤형억제전략에 활용 방안, 동북아 지역 역내 국가들의 저위력 핵무기 개발 시도 및 핵전략 변화 전망 등에 관한 연구를 기대해 본다.

\section{Acknowledgements}

We would like to thank Editage (www.editage.co.kr) for English language editing.

\section{Declaration of Conflicting Interests}

The author(s) declared no potential conflicts of interest with respect to the research, authorship, and/or publication of this article. 
A study on the development and implications of low-yield nuclear weapons, focusing on ... / Sungjoon Moon 129

\section{Reference}

Ahn, M. S. (2019). The Kim Jong-un Regimes Strategy of Nuclear Warhead and Long-range Missile. Journal of Northeast Asian Studies, 82, 173-190. UCI : G704-001454.2017.22.1.006

Brodie, B. (1959). Strategy in the Missile Age. Naval War College Review, 12(9), 8. https://doi.org/10.1515/9781400875108

COCHAIRS, T. F. (2021). Preventing Nuclear Proliferation and Reassuring America's Allies: Report from the Task Force on US Allies and Nuclear Weapons Proliferation. https://www.jstor.org/stable/resrep29698

Cummings, A. (2020). (2020). High Speed, low-Yield: A US Dual-Use Hypersonic Weapon. https://warontherocks.com/2020/09/high-speed-low-yield-a-u-u-dual-use-hypersonic-w eapon/

Haffa Jr, R. P. (2018). The future of conventional deterrence: Strategies for great power competition. Strategic Studies Quarterly, 12(4), 94-115. https:/www.jstor.org/stable/ 26533617

Harkavy, R. E. (1998). Triangular or indirect deterrence/compellence: Something new in deterrence theory?. Comparative Strategy, 17(1), 63-81. https://doi.org/10.1080/ 01495939808403132

Hwang, H. P. (2021). North Korea's Evolving Nuclear Posture: Strategic Implications of Pyongyang's Tactical Nuclear Weapons Development. The Quarterly Journal of Defense Policy Studies, 37(3), 7-43. https://doi.org/10.22883/jdps.2021.37.3.001

Hwang, I. D. (2021). Common Pattern of Nuclear Doctrine Evolutions and North Korea's Recent Concept of Nuclear Escalation. National Strategy, 27(3), 5-26. https://doi.org/10.35390/ sejong.27.3.202108.001

Hwang, J. W. (2021). U.S. Extended Deterrence to Korea in Decline?: The Evolution of Extended Deterrence and Reassessment of Its Credibility. National Strategy, 27(3), 27-52. https://doi.org/10.35390/sejong.27.3.202108.002

Lee, G. W. (2018, March). Nuclear Strategy in the Modern Era: : Regional Nuclear Powers and International Conflict by Vipin Narang. Strategic Studies, 25(1), 263-277. https://www.dbpia.co.kr/pdf/pdfView?nodeId=NODE07408523

Lieber, K. A., \& Press, D. G. (2017). The new era of counterforce: Technological change and the future of nuclear deterrence. International Security, 41(4), 9-49. https://doi.org/10.1162/ 
ISEC_a_00273

Narang, V. (2014). Nuclear Strategy in the Modern Era: Regional Powers and International Conflict. N.J.: Princeton University Press. https://press.princeton.edu/books/paperback/ 9780691159836/nuclear-strategy-in-the-modern-era

Park, J. W. (2021). Development and Implications of the U.S. Low-Yield Nuclear Weapons to Enhance the Reliability of Extended Deterrence. Korea and Global Affairs, 5(5), 179-198. https://doi.org/10.22718/kga.2021.5.5.179

Shin, D. H. (2018). North Korea's Nuclear Strategy analyzed with Nuclear Deterrrence Theory. Korean Journal of Military Art and Science, 74(1), 145-164. https://doi.org/10.31066/ kjmas.2018.74.1.006

Snyder, G. H. (1961). Deterrence and Defense: Toward a Theory of National Security. N.J.: Princeton University Press.

Sohn, H. B. (2015). The ROK-US Alliance in the Post-Cold War Era: Alliance Management for strengthening its Extended Deterrence. The studies of New Security Challenges, 187, 41-72. UCI : G704-SER000010556.2015..187.005

Wohlstetter, A. (1959). The delicate balance of terror. Survival: Global Polities and Strategy, 1(1), 8 - 17. https://doi.org/10.1080/00396335908440116 
IDAP 광운대학교 방위사업연국소

2021, Vol. 4, No. 3, 113-131.

https://doi.org/10.37944/jams.v4i3.132

\section{억제이론을 중심으로 저위력 핵무기 개발 및 함의에 관한 연구}

문성준*

\section{국문초목}

본 연구의 목적은 미국 트럼프 행정부에서 시작한 저위력 핵무기의 개발 현황과 저위력 핵무기의 기 술적 특성을 살펴보고 현 바이든 행정부에서의 개발 추세를 전망한 후에 한미 '맞춤형 억제전략(TDR: Tailored Deterrence Strategy)'에 미치는 전략적 함의를 분석하고 한국의 향후 대응방안을 모색하는 것 이다.

억제이론 측면에서 저위력 핵무기는 거부적 억제(deterrence by denial)와 보복적 억제(deterrence by retaliation)를 동시에 확대할 수 있는 수단으로 평가받고 있다. 또한 저위력 핵무기는 '사용가능한 핵무 기'라는 점에서 억제의 $3 \mathrm{C}$ 요소인 사용가능한 능력(capability), 핵보복 의지와 가능성의 의사전달 (communication), 이러한 능력과 의지에 대한 신뢰성(credibility)을 구비하여 억제의 수단으로서 활용 가 치가 높다고 평가할 수 있다.

북한의 핵 - 미사일 능력이 고도화되었고 미국이 저위력 핵무기 개발 및 배치를 하고 있는 시점에 억 제 및 대응 측면에서 몇 가지 제안을 하고자 한다. 첫째, 2021년 들어 북한의 전술핵 역량 강화방침은 필연적으로 저위력 핵무기 개발의 기술적 특성과 매우 밀접한 연관이 있어 이를 연계하여 평가하고 대비해야 할 것이다. 둘째, 미국의 확장억제전략 측면에서 바이든 행정부의 핵전략을 이해하고 2022년 초에 공개되는 5 차 NPR을 토대로 긴밀히 협의해 나갈 필요가 있다. 셋째, 한미 맞춤형억제전략의 신뢰 성를 보장하기 위해 저위력 핵무기를 포함하는 지역 차원의 ‘다자 억제 방안’을 진지하게 검토해야 할 것이다.

주제어 : 저위력 핵무기, 삼각억제, 억제 $3 \mathrm{C}$ 요소, 맞춤형억제전략, 다자 억제 방안 
